\title{
Characterization and Quality Assessment of Potential Indigenous Mango (Mangifera indica L.) Cultivars of Coastal Districts in Andhra Pradesh, India by Bio-Chemical Markers
}

\author{
A. Himabindu*, D. Srihari, M. Rajasekhar, V. Sudhavani, \\ P. Subbarammamma and K. Uma Krishna
}

Horticultural Research Station, Dr. Y.S.R Horticultural University, Venkataramannagudem, West Godavari, Andhra Pradesh - 534101, India

*Corresponding author

\section{A B S T R A C T}

\begin{tabular}{|l|}
\hline Ke y w or d s \\
Carotene, \\
Characterization, Mango, \\
Bio-chemical, Total \\
phenols
\end{tabular}

\section{Introduction}

Mango (Mangifera indica L.) is an important member of the family Anacardiaceae in order Sapindales and is the most important fruit crop in India having a great cultural, socioeconomic and religious significance since ancient times. It is said to be originated in the Indo-Burma (Myanmar) region (De Candolle, 1904). By virtue of its excellent flavor, delicious taste, attractive color, fruit quality with richness in vitamins and minerals, accessibility to common man, liking by the masses, mango has been assigned the status of the 'King of the fruits' in the tropical world and it is the 'National Fruit of India'. In India mango ranks first in terms of area with 2.50 million ha, second in respect of production with 18.00 million tonnes and with a productivity of 7.2 million tonnes/ha, while Andhra Pradesh ranks second in terms of area with 0.30 million ha, second in respect to production of 2.70 million tonnes and with a productivity of 9.0 million tonnes/ha (NHB, 2013). Andhra Pradesh is considered as a centre of diversity for mango with a rich 
diversity of named local cultivars and unnamed local land races. Mango is considered to be an allopolyploid, most probably amphidiploid and outbreeding species having chromosome number $2 n=40$ (Mukherjee, 1950). It is highly heterozygous as performance varies with the climate which resulted in a high level of genetic diversity. Further, confusion exists in the nomenclature of mangoes due to different local names for the same variety. Knowledge of the magnitude of genetic variation among the land races of fruit characteristics is important for development of new varieties of mango with improved quality is the engine of market demand. The assessment of diversity based on morphological parameters has been often constrained by lack of adequate data on distinguishable morphological characters and confusion due to the wide variations for a particular trait in a given population. Though, morphological markers have been in use to assess the genetic diversity, they had limited application in breeding as they are few in number and phenotype is influenced by environment so, bio-chemical markers offer greater diversity as compared to the morphological markers. The objective of the study was to evaluate fruit bio-chemical markers of 34 indigenous mango cultivars and to isolate and identify the superior genotypes for future breeding programmes.

\section{Materials and Methods}

The present study was conducted to study the performance of mango cultivars of coastal districts in Andhra Pradesh at Horticultural Research Station, Venkataramannagudem during the subsequent years of 2013 and 2014. A well- planned germplasm collection survey based on diversity richness was conducted in coastal districts of Andhra Pradesh which includes Horticultural Research Station and private owned mango orchards. Random sampling strategy was followed for collection of samples. Three plants in each cultivar were taken as sample size. The experimental material consists of 34 indigenous mango cultivars and variants within them obtained from the coastal districts of Andhra Pradesh. The indigenous mango cultivars used are:

Banganapalli - 1

Banganapalli - 2

Banganapalli - 3

Banglora - 1

Banglora - 2

Baramasi

Cherukurasam

Chinnarasam

Chinna Suvarnarekha

Elamandala

Hyder

Imampasand

Jalal

Jehangir

Kolanka Goa

Kottapalli Kobbari

Kowsuri Pasand

Nalla Andrews

Nalla Rasalu

Navaneetam

Nuzividu Tiyya Mamidi

Nuzividu Rasalu

Panchadara Kalasa

Pandurivari Mamidi

Paparao Goa

Peddarasam

Panukula Mamidi

Royal Special

Rajamanu

Sora Mamidi

Suvarnarekha

Tella Gulabi

Tella Rasalu

Rajamamidi

Five fruits of each cultivar were taken per replication for evaluating fruit bio-chemical characters. Longitudinal slices of fruit pulp were used to extract juice with the help of 
standard commercial juicer. The juice was extracted from each sample and homogenized to study the bio-chemical parameters.

\section{Total soluble solids ( $\left.{ }^{\mathrm{O}} \mathrm{Brix}\right)$}

The percentage of total soluble solids (TSS) was determined by using ERMA hand refractrometer by placing a drop of filtered juice on the prism of the refractrometer and observed the coincidence of shadow of the sample with the reading on the scale and expressed as ${ }^{\mathrm{O}}$ Brix. Before taking the reading, the refractrometer was tested for its error with distilled water, corrected accordingly and TSS content was recorded (Ranganna, 1986).

\section{Titratable acidity (\%)}

The titratable acidity was determined by taking $10 \mathrm{ml}$ of homogenized sample and volume was made up to $100 \mathrm{ml}$ with distilled water in a volumetric flask. The contents were filtered through Whatman No.1 filter paper.

An aliquot of $10 \mathrm{ml}$ was taken in $250 \mathrm{ml}$ conical flask for titration against $0.1 \mathrm{~N} \mathrm{NaOH}$ by using phenolphthalein as an indicator. The turn of aliquot to light pink colour which persists for 15 seconds was considered as an end point and the titratable acidity was estimated in terms of per cent citric acid (Ranganna, 1986).

Factor for acidity $=1 \mathrm{ml}$ of $\mathrm{N} / 10 \mathrm{NaOH}=$ $0.0064 \mathrm{~g}$ of citric acid

Titer value $\mathrm{x}$ Normality of $\mathrm{NaOH} \mathrm{x}$ $0.0064 \times 100$ Acidity $(\%)=$

Volume of aliquot taken (ml)

\section{TSS: acid ratio}

Total soluble solids/Acidity ratio (TSS: acid ratio) was calculated by dividing the value of
TSS with that of corresponding titratable acidity.

\section{Reducing sugars (\%)}

Reducing sugars were determined by Lane and Eyon method (AOAC, 1965). Twenty five ml of fruit juice was taken and transferred to 250 $\mathrm{ml}$ volumetric flask. Two $\mathrm{ml}$ of lead acetate solution (45\%) was added to flask for precipitation of colloidal matter. Potassium oxalate $(22 \%)$ of $2 \mathrm{ml}$ was added in this solution to precipitate the lead and the volume made up to $250 \mathrm{ml}$ using distilled water.

The contents were then filtered through Whatman No. 1 filter paper after testing a little of filtrate for its freedom from lead by adding a drop of potassium oxalate. Reducing sugars in the lead free solution was taken in burette and titrated against $10 \mathrm{ml}$ of standard Fehling's solution mixture of A and B (1: 1) by using methylene blue as an indicator till the end point was indicated by the formation of brick red precipitate.

The titration was carried out by keeping the Fehling's solution boiling on the heating mantle. The results were expressed as per cent reducing sugar by using the following formula:

Factor x Volume made up x 100 Reducing sugars $(\%)=$--------------------Titer value $\mathrm{x}$ Weight of the sample $10 \mathrm{ml}$ of Fehling solution $=0.05$ glucose .

\section{Total sugars $(\%)$}

Total sugars were determined as procedure described by Lane and Eyon method (AOAC, 1965). A quantity of $50 \mathrm{ml}$ lead free filtrate was taken in a $100 \mathrm{ml}$ volumetric flask and to it $5 \mathrm{ml}$ of concentrated $\mathrm{HCl}$ was added, mixed well and then kept for 24 hours at room 
temperature. Acid was then neutralized with $\mathrm{NaOH}$ by using a drop of phenolphthalein as an indicator till the pink color persisted for at least few seconds. Then volume was made up to $100 \mathrm{ml}$. Total sugars were then estimated by taking this solution in a burette and titrating it against standard Fehling's solution mixture of $\mathrm{A}$ and B (1:1) by using methylene blue as an indicator and taking brick red colour as an end point. The per cent total sugars were calculated by using the following formula:

Factor x Volume made up x 100 Total sugars $(\%)=$

Titer value $\mathrm{x}$ Weight of the sample

\section{Non-reducing sugars (\%)}

Non-reducing sugars in juice was obtained by subtracting reducing sugars from total sugars.

Non-reducing sugars $(\%)=$ Total sugars Reducing sugars

\section{Ascorbic acid (mg/100ml)}

$10 \mathrm{ml}$ of juice was blended with metaphosphoric acid $\left(3 \% \mathrm{HPO}_{3}\right)$ and volume was made up to $100 \mathrm{ml}$ with $\mathrm{HPO}_{3}(3 \%)$. The content after shaking well was filtered through Whatman No.1 filter paper. Ten $\mathrm{ml}$ of filtrate was titrated against 2,6 dichlorophenolindophenol dye until light pink colour was observed (AOAC, 1965).

Titer value $\mathrm{x}$ Dye factor $\mathrm{x}$ Volume made up x 100 Ascorbic acid $(\mathrm{mg} / 100 \mathrm{ml})=$

Volume of the sample $\mathrm{x}$ Volume of aliquot taken

\section{$\beta$ - Carotene $(\mu \mathrm{g} / \mathbf{1 0 0 g})$}

Five grams of fresh sample was weighed with the help of electronic balance and crushed with $10-15 \mathrm{ml}$ of acetone and a few crystals of anhydrous sodium sulphate, with the help of mortar and pestle. Decant the supernatant into a beaker. Repeat the process twice and transfer the combined supernatant sample into a separatory funnel, $10-15 \mathrm{ml}$ petroleum ether was added and mixed thoroughly.

Two layers were separated out on standing. Discard the lower layer and collect the upper layer into a $100 \mathrm{ml}$ volumetric flask, made up the volume to $100 \mathrm{ml}$ with petroleum ether and record the optical density at $425 \mathrm{~nm}$ by using Thermo Evolution 201 Model spectrophotometer as petroleum ether as blank (Srivastava and Sanjeev, 2014).

$$
\begin{aligned}
& \text { B-carotene }(\mu \mathrm{g} / 100 \mathrm{~g})= \\
& \text { Weight of sample } \times 560 \times 1000
\end{aligned}
$$

\section{Total phenols (mg of gallic acid /100g)}

Phenols were estimated according to the procedure given by Malik and Singh (1980). One gram of sample was extracted with $10 \mathrm{ml}$ of 80 per cent methanol. The homogenated extracts were centrifuged at 10,000 rpm for 20 min by using "5430 R Eppendorf” centrifuge and the supernatant saved was evaporated to dryness in a water bath. The residue was dissolved in $5 \mathrm{ml}$ of distilled water and $0.5 \mathrm{ml}$ of Folin -Ciocalteau's reagent $(1 \mathrm{~N})$ was added.

To that, $2 \mathrm{ml}$ of sodium carbonate (20\%) solution was added and after mixing thoroughly the tubes were placed in boiling water for one minute, cooled and the absorbance was measured at $650 \mathrm{~nm}$ by using Thermo Evolution 201 model spectrophotometer.

Standard curve was drawn by using gallic acid as standard. Different concentrations of gallic acid were prepared and O.D was read at 650 nm. The concentration of samples was calculated based on the standard curve. 
O.D $\times$ Factor $\times$ Volume made up $\times 100$ Total Phenols $(\mathrm{mg}$ of GA/100g) $=$

Aliquot taken $\times$ Weight of the sample $\times 1000$

\section{Results and Discussion}

The data recorded on fruit bio-chemical characters of mango were subjected to statistical analysis. Significant differences were observed among the cultivars for all the characters studied. TSS content ranged from $15.05^{\circ}$ Brix to $25.44^{\circ}$ Brix with a mean of 18.27 Brix (Table 1 and Fig.1). TSS was observed to be highest in cultivar Cherukurasam $\left(25.44^{\circ}\right.$ Brix $)$ followed by Elamandala $\left(23.55^{\circ}\right.$ Brix $), \quad$ Banganapalli-1 $\left(21.27^{\circ}\right.$ Brix) which was on par with Baramasi (21.04 ${ }^{\circ}$ Brix). Lowest TSS was observed in cultivar Sora Mamidi (15.05 Brix) followed by Navaneetham $\left(15.31^{\circ}\right.$ Brix $)$, which was on par with Kowsuri Pasand $\left(15.58^{\circ}\right.$ Brix) and Banglora-2 (15.69 $\left.{ }^{\circ} \mathrm{Brix}\right)$. Similar trend in TSS content of mango cultivars is reported by Kulkarni and Rameshwar (1981) in which TSS ranged from $13.1 \%$ to $27 \%$ and Hameedunissa Begum et al., (2013) reported similar values of TSS in Banganapalli which ranged from 15 to $22^{\circ} \mathrm{Brix}$. The range of titratable acidity (Table 1 ) among the mango cultivars studied in the present investigation was 0.14 to $1.19 \%$ with a mean of $0.30 \%$. The cultivar Royal Special recorded the highest titratable acidity (1.19\%) followed by Baramasi (0.73\%), Kottapalli Kobbari (0.33) while, the cultivar Banganapalli-1 (0.14\%) recorded the lowest titratable acidity which was on par with Cherukurasam and Panchadara Kalasa $(0.17 \%)$. Similarly the lowest titratable acidity content of $0.14 \%$ in mango cultivars is reported by Rathor (2005). Maximum TSS: acid ratio was noticed in Banganapalli-1 (155.95) followed by Cherukurasam (147.91), Elamandala (135.23), Panchadara Kalasa (117.33), Suvarnarekha (113.50) and Panukula Mamidi (104.32) while, minimum TSS: acid ratio was noticed in Royal special (17.34) followed by Rajamamidi (49.09) and Jehangir (52.08). Further, Sora Mamidi (49.84) and Kowsuri Pasand (50.38) were on par with Rajamamidi. Similarly high TSS: acid ratio of 162.70 in mango cultivars is reported by Rathor (2005). With respect to important bio-chemical markers namely TSS, titratable acidity as well as TSS: acid ratio, Banganapalli-1, Cherukurasam, Elamandala, Panchadara Kalasa, Panukula Mamidi and Suvarnarekha were found to be promising mango cultivars. These cultivars have TSS amounting to $20^{\circ}$ Brix or even more, which is a desirable character, and they had less acidity and high sugar: acid ratio can be used as good donors in future hybridization programme to evolve superior varieties and hybrids. The mean per cent of total sugars was $12.27 \%$, while it ranged from 8.82 to $21.51 \%$ (Table 2 and Fig.1). Highest per cent of total sugars were recorded in the cultivars, Cherkurasam $(21.51 \%)$ followed by Banganapalli-1 (18.28\%), Banganapalli-3 (16.45\%) and Suvarnarekha (14.68\%). Further, the cultivars Tella Rasalu (14.24\%) and Tella Gulabi $(14.22 \%)$ were on par with Suvarnarekha. Lowest per cent of total sugars were recorded in the cultivars, Nuzividu Rasalu $(8.82 \%)$ which was on par with Banglora-2 $(9.10 \%)$ followed by Navaneetham (9.68\%). Similar results with regard to total sugars were earlier reported by Rathor (2005) in mango. The mango cultivars differed significantly in reducing sugar content while, it ranged from 2.63 to $6.44 \%$ with a mean of $4.34 \%$ (Table $2)$. The highest reducing sugar content was found in the cultivar Banganapalli-1 (6.44\%) followed by Banganapalli-3 (6.30\%), Cherukurasam $(6.11 \%)$ and Elamandala $(5.73 \%)$. The lowest reducing sugar content was found in Banglora-2 (2.63\%) followed by Nuzividu Rasalu (3.03\%). Similar observations are reported by Simi (2006) with respect to average reducing sugars $(3.11 \%)$ in mango. 
Table.1 Mean performance of mango cultivars for TSS, titratable acidity and TSS: acid ratio

\begin{tabular}{|c|c|c|c|c|c|c|c|c|c|c|}
\hline \multirow[t]{2}{*}{$\begin{array}{l}\text { S. } \\
\text { No }\end{array}$} & \multirow[t]{2}{*}{ Name of the Cultivar } & \multicolumn{3}{|c|}{ TSS ( ${ }^{\circ}$ Brix) } & \multicolumn{3}{|c|}{$\begin{array}{c}\text { Titratable Acidity } \\
(\%)\end{array}$} & \multicolumn{3}{|c|}{ TSS: acid Ratio } \\
\hline & & 2013 & 2014 & Mean & 2013 & 2014 & Mean & 2013 & 2014 & Mean \\
\hline 1 & Banganapalli - 1 & 21.12 & 21.43 & 21.27 & 0.15 & 0.13 & 0.14 & 143.56 & 168.34 & 155.95 \\
\hline 2 & Banganapalli - 2 & 17.42 & 16.87 & 17.15 & 0.33 & 0.32 & 0.32 & 53.11 & 53.10 & 53.10 \\
\hline 3 & Banganapalli - 3 & 19.75 & 20.98 & 20.36 & 0.25 & 0.22 & 0.23 & 80.18 & 97.20 & 88.69 \\
\hline 4 & Banglora -1 & 16.67 & 16.55 & 16.61 & 0.23 & 0.24 & 0.23 & 74.00 & 69.94 & 71.97 \\
\hline 5 & Banglora -2 & 15.26 & 16.12 & 15.69 & 0.28 & 0.24 & 0.26 & 55.11 & 67.59 & 61.35 \\
\hline 6 & Baramasi & 21.65 & 20.43 & 21.04 & 0.75 & 0.71 & 0.73 & 28.94 & 28.83 & 28.88 \\
\hline 7 & Cherukurasam & 26.34 & 24.55 & 25.44 & 0.17 & 0.18 & 0.17 & 155.28 & 140.55 & 147.91 \\
\hline 8 & Chinnarasam & 16.86 & 18.89 & 17.88 & 0.25 & 0.21 & 0.23 & 67.68 & 91.47 & 79.57 \\
\hline 9 & Chinna Suvarnarekha & 15.91 & 16.76 & 16.33 & 0.26 & 0.22 & 0.24 & 62.32 & 77.67 & 69.99 \\
\hline 10 & Elamandala & 24.23 & 22.87 & 23.55 & 0.18 & 0.18 & 0.18 & 141.59 & 128.87 & 135.23 \\
\hline 11 & Hyder & 18.78 & 17.54 & 18.16 & 0.27 & 0.29 & 0.28 & 70.67 & 60.70 & 65.68 \\
\hline 12 & Imampasand & 18.82 & 17.95 & 18.39 & 0.26 & 0.27 & 0.26 & 74.10 & 66.56 & 70.33 \\
\hline 13 & Jalal & 16.67 & 16.23 & 16.45 & 0.30 & 0.31 & 0.31 & 56.62 & 52.55 & 54.58 \\
\hline 14 & Jehangir & 15.89 & 15.76 & & 0.30 & 0.31 & 0.31 & 53.24 & 50.92 & 52.08 \\
\hline 15 & Kolanka Goa & 17.21 & 16.89 & 17.05 & 0.29 & 0.27 & 0.28 & 59.72 & 62.88 & 61.30 \\
\hline 16 & Kottapalli Kobbari & 17.72 & 17.43 & & 0.33 & 0.33 & 0.33 & 53.98 & 52.98 & 53.48 \\
\hline 17 & Kowsuri Pasand & 15.84 & 15.32 & 15.58 & 0.31 & 0.31 & 0.31 & 51.18 & 49.59 & 50.38 \\
\hline 18 & Nalla Andrews & 17.42 & 18.54 & & 0.32 & 0.29 & 0.31 & 54.59 & 63.97 & 59.28 \\
\hline 19 & Nalla Rasalu & 16.23 & 16.33 & 16.28 & 0.32 & 0.29 & 0.31 & 50.83 & 56.53 & 53.68 \\
\hline 20 & Navaneetam & 14.29 & 16.32 & & 0.27 & 0.25 & 0.26 & 53.89 & 65.36 & 59.62 \\
\hline 21 & $\begin{array}{l}\text { Nuzividu Tiyya } \\
\text { Mamidi }\end{array}$ & 16.63 & 18.11 & 17.37 & 0.28 & 0.27 & 0.28 & 59.85 & 67.20 & 63.53 \\
\hline 22 & Nuzividu Rasalu & 16.48 & 15.66 & & 0.29 & 0.30 & 0.30 & 56.86 & 52.23 & 54.54 \\
\hline 23 & Panchadara Kalasa & 20.64 & 19.44 & 20.04 & 0.17 & 0.18 & 0.17 & 125.45 & 109.21 & 117.33 \\
\hline 24 & Pandurivari Mamidi & 19.89 & 17.99 & 18.94 & 0.21 & 0.22 & 0.22 & 96.17 & 83.10 & 89.63 \\
\hline 25 & Paparao Goa & 19.43 & 18.62 & 19.02 & 0.23 & 0.25 & 0.24 & 86.34 & 74.84 & 80.59 \\
\hline 26 & Peddarasam & 18.86 & 20.56 & 19.71 & 0.26 & 0.23 & 0.25 & 73.71 & 89.95 & 81.83 \\
\hline 27 & Panukula Mamidi & 19.88 & 20.75 & 20.32 & 0.21 & 0.19 & 0.20 & 96.82 & 111.83 & 104.32 \\
\hline 28 & Royal Special & 21.24 & 20.12 & 20.68 & 1.23 & 1.16 & 1.19 & 17.31 & 17.37 & 17.34 \\
\hline 29 & Rajamanu & 17.12 & 18.53 & 17.83 & 0.30 & 0.26 & 0.28 & 57.25 & 73.84 & 65.54 \\
\hline 30 & Sora Mamidi & 14.98 & 15.12 & 15.05 & 0.31 & 0.30 & 0.31 & 48.97 & 50.72 & 49.84 \\
\hline 31 & Suvarnarekha & 20.86 & 19.45 & 20.16 & 0.18 & 0.18 & 0.18 & 118.75 & 108.26 & 113.50 \\
\hline 32 & Tella Gulabi & 18.43 & 17.98 & 18.20 & 0.27 & 0.28 & 0.28 & 69.14 & 65.53 & 67.33 \\
\hline 33 & Tella Rasalu & 18.42 & 17.98 & 18.20 & 0.27 & 0.27 & 0.27 & 68.52 & 66.98 & 67.75 \\
\hline 34 & Rajamamidi & 15.57 & 15.64 & 15.60 & 0.33 & 0.31 & 0.32 & 47.35 & 50.84 & 49.09 \\
\hline & Mean & 18.31 & 18.23 & 18.27 & 0.30 & 0.29 & 0.30 & 72.44 & 74.34 & 73.39 \\
\hline & SEm \pm & & & 0.28 & & & 0.01 & & & 0.94 \\
\hline & C.D 5\% & & & 0.77 & & & 0.03 & & & 2.62 \\
\hline & C.D $1 \%$ & & & 1.01 & & & 0.04 & & & 3.46 \\
\hline
\end{tabular}


Table.2 Mean performance of mango cultivars for total, reducing and non-reducing sugar content

\begin{tabular}{|c|c|c|c|c|c|c|c|c|c|c|}
\hline \multirow[t]{2}{*}{$\begin{array}{l}\text { S. } \\
\text { No }\end{array}$} & \multirow[t]{2}{*}{ Name of the Cultivar } & \multicolumn{3}{|c|}{ Total Sugars (\%) } & \multicolumn{3}{|c|}{$\begin{array}{c}\text { Reducing Sugars } \\
(\%)\end{array}$} & \multicolumn{3}{|c|}{$\begin{array}{c}\text { Non-reducing Sugars } \\
(\%)\end{array}$} \\
\hline & & 2013 & 2014 & Mean & 2013 & 2014 & Mean & 2013 & 2014 & Mean \\
\hline 1 & Banganapalli - 1 & 18.12 & 18.44 & 18.28 & 6.34 & 6.54 & 6.44 & 11.78 & 11.90 & 11.84 \\
\hline 2 & Banganapalli - 2 & 11.50 & 11.32 & 11.41 & 4.32 & 4.01 & 4.16 & 7.18 & 7.31 & 7.24 \\
\hline 3 & Banganapalli - 3 & 16.12 & 16.78 & 16.45 & 6.29 & 6.31 & 6.30 & 9.83 & 10.47 & 10.15 \\
\hline 4 & Banglora -1 & 11.16 & 10.98 & 11.07 & 4.13 & 4.00 & 4.07 & 7.03 & 6.98 & 7.01 \\
\hline 5 & Banglora -2 & 8.32 & 9.89 & 9.10 & 2.02 & 3.24 & 2.63 & 6.30 & 6.65 & 6.48 \\
\hline 6 & Baramasi & 12.86 & 11.86 & 12.36 & 4.94 & 4.54 & 4.74 & 7.92 & 7.32 & 7.62 \\
\hline 7 & Cherukurasam & 22.01 & 21.01 & 21.51 & 6.25 & 5.98 & 6.11 & 15.76 & 15.03 & 15.39 \\
\hline 8 & Chinnarasam & 11.63 & 12.23 & 11.93 & 4.12 & 4.87 & 4.49 & 7.51 & 7.36 & 7.43 \\
\hline 9 & Chinna Suvarnarekha & 8.48 & 11.69 & 10.09 & 3.04 & 4.32 & 3.68 & 5.44 & 7.37 & 6.40 \\
\hline 10 & Elamandala & 17.23 & 16.75 & 16.99 & 5.82 & 5.64 & 5.73 & 11.41 & 11.11 & 11.26 \\
\hline 11 & Hyder & 10.69 & 9.87 & 10.28 & 4.12 & 4.01 & 4.07 & 6.57 & 5.86 & 6.22 \\
\hline 12 & Imampasand & 12.69 & 12.12 & 12.40 & 4.56 & 4.32 & 4.44 & 8.13 & 7.80 & 7.97 \\
\hline 13 & Jalal & 10.83 & 10.42 & 10.63 & 4.12 & 3.98 & 4.05 & 6.71 & 6.44 & 6.57 \\
\hline 14 & Jehangir & 10.87 & 11.43 & 11.15 & 4.01 & 3.76 & 3.88 & 6.86 & 7.67 & 7.27 \\
\hline 15 & Kolanka Goa & 12.07 & 11.65 & 11.86 & 4.44 & 4.12 & 4.28 & 7.63 & 7.53 & 7.58 \\
\hline 16 & Kottapalli Kobbari & 10.61 & 10.03 & 10.32 & 3.13 & 3.01 & 3.07 & 7.48 & 7.02 & 7.25 \\
\hline 17 & Kowsuri Pasand & 11.57 & 11.12 & 11.35 & 4.06 & 3.97 & 4.01 & 7.51 & 7.15 & 7.33 \\
\hline 18 & Nalla Andrews & 10.96 & 11.65 & 11.31 & 3.67 & 4.21 & 3.94 & 7.29 & 7.44 & 7.36 \\
\hline 19 & Nalla Rasalu & 9.32 & 10.97 & 10.15 & 2.84 & 3.76 & 3.30 & 6.48 & 7.21 & 6.85 \\
\hline 20 & Navaneetam & 8.48 & 10.87 & 9.68 & 2.96 & 3.57 & 3.26 & 5.52 & 7.30 & 6.41 \\
\hline 21 & Nuzividu Tiyya Mamidi & 9.76 & 12.32 & 11.04 & 3.09 & 3.97 & 3.53 & 6.67 & 8.35 & 7.51 \\
\hline 22 & Nuzividu Rasalu & 8.77 & 8.87 & 8.82 & 3.04 & 3.01 & 3.03 & 5.73 & 5.86 & 5.80 \\
\hline 23 & Panchadara Kalasa & 12.94 & 11.61 & 12.27 & 4.43 & 4.21 & 4.32 & 8.51 & 7.40 & 7.95 \\
\hline 24 & Pandurivari Mamidi & 12.67 & 12.12 & 12.39 & 4.71 & 4.53 & 4.62 & 7.96 & 7.59 & 7.78 \\
\hline 25 & Paparao Goa & 13.58 & 13.54 & 13.56 & 4.56 & 4.39 & 4.47 & 9.02 & 9.15 & 9.09 \\
\hline 26 & Peddarasam & 10.19 & 13.02 & 11.60 & 3.95 & 4.71 & 4.33 & 6.24 & 8.31 & 7.28 \\
\hline 27 & Panukula Mamidi & 11.09 & 12.87 & 11.98 & 3.77 & 4.39 & 4.08 & 7.32 & 8.48 & 7.90 \\
\hline 28 & Royal Special & 12.12 & 11.64 & 11.88 & 4.59 & 4.35 & 4.47 & 7.53 & 7.29 & 7.41 \\
\hline 29 & Rajamanu & 10.31 & 11.76 & 11.03 & 4.68 & 4.91 & 4.80 & 5.63 & 6.85 & 6.24 \\
\hline 30 & Sora Mamidi & 9.93 & 9.87 & 9.90 & 3.12 & 3.00 & 3.06 & 6.81 & 6.87 & 6.84 \\
\hline 31 & Suvarnarekha & 15.58 & 13.78 & 14.68 & 5.27 & 5.12 & 5.19 & 10.31 & 8.66 & 9.49 \\
\hline 32 & Tella Gulabi & 14.57 & 13.87 & 14.22 & 5.13 & 5.07 & 5.10 & 9.44 & 8.80 & 9.12 \\
\hline 33 & Tella Rasalu & 13.64 & 14.85 & 14.24 & 5.66 & 5.28 & 5.47 & 7.98 & 9.57 & 8.77 \\
\hline 34 & Rajamamidi & 10.97 & 11.74 & 11.35 & 4.44 & 4.21 & 4.32 & 6.53 & 7.53 & 7.03 \\
\hline & Mean & 12.11 & 12.44 & 12.27 & 4.28 & 4.39 & 4.34 & 7.82 & 8.05 & 7.94 \\
\hline & SEm \pm & & & 0.29 & & & 0.13 & & & 0.22 \\
\hline & C.D 5\% & & & 0.80 & & & 0.35 & & & 0.62 \\
\hline & C.D $1 \%$ & & & 1.05 & & & 0.46 & & & 0.81 \\
\hline
\end{tabular}


Table.3 Mean performance of mango cultivars for ascorbic acid,

$\beta$-carotene and total phenol content

\begin{tabular}{|c|c|c|c|c|c|c|c|c|c|c|}
\hline \multirow[t]{2}{*}{$\begin{array}{l}\text { S. } \\
\text { No }\end{array}$} & \multirow[t]{2}{*}{ Name of the Cultivar } & \multicolumn{3}{|c|}{$\begin{array}{l}\text { Ascorbic Acid } \\
\text { (mg/100g) }\end{array}$} & \multicolumn{3}{|c|}{$\beta$ - Carotene $(\mu \mathrm{g} / 100 \mathrm{~g})$} & \multicolumn{3}{|c|}{$\begin{array}{l}\text { Total Phenols (mg } \\
\text { of GA/ 100g) }\end{array}$} \\
\hline & & 2013 & 2014 & Mean & 2013 & 2014 & Mean & 2013 & 2014 & Mean \\
\hline 1 & Banganapalli - 1 & 28.18 & 25.76 & 26.97 & 1689.98 & 1650.32 & 1670.15 & 35.99 & 38.62 & 37.31 \\
\hline 2 & Banganapalli - 2 & 27.45 & 26.76 & 27.10 & 923.45 & 910.21 & 916.83 & 73.95 & 71.21 & 72.58 \\
\hline 3 & Banganapalli - 3 & 27.54 & 30.87 & 29.20 & 1012.67 & 1000.21 & 1006.44 & 48.60 & 49.54 & 49.07 \\
\hline 4 & Banglora - 1 & 25.25 & 23.43 & 24.34 & 1223.43 & 1211.59 & 1217.51 & 53.31 & 50.93 & 52.12 \\
\hline 5 & Banglora - 2 & 32.50 & 35.76 & 34.13 & 765.21 & 738.90 & 752.05 & 64.65 & 65.73 & 65.19 \\
\hline 6 & Baramasi & 87.25 & 89.54 & 88.40 & 706.87 & 699.61 & 703.24 & 85.90 & 83.29 & 84.60 \\
\hline 7 & Cherukurasam & 27.25 & 26.92 & 27.08 & 1321.76 & 1293.81 & 1307.79 & 37.78 & 32.71 & 35.24 \\
\hline 8 & Chinnarasam & 37.32 & 40.71 & 39.01 & 1708.76 & 1651.02 & 1679.89 & 62.35 & 55.83 & 59.09 \\
\hline 9 & Chinna Suvarnarekha & 28.54 & 34.70 & 31.62 & 791.34 & 740.37 & 765.85 & 98.12 & 84.74 & 91.43 \\
\hline 10 & Elamandala & 20.54 & 23.74 & & 821.34 & 801.29 & 811.32 & 39.76 & 38.88 & 39.32 \\
\hline 11 & Hyder & 22.50 & 28.63 & 25.57 & 1698.34 & 1638.65 & 1668.49 & 48.22 & 52.37 & 50.29 \\
\hline 12 & Imam & 29.57 & 36.83 & & 893.56 & & & 56.32 & 57.83 & 57.08 \\
\hline 13 & Jalal & 23.75 & 28.95 & 26.35 & 1193.34 & 1109.36 & 1151.35 & 50.97 & 51.92 & 51.44 \\
\hline 14 & Jehan & 22.75 & 29.29 & 26.02 & 816.78 & 793.45 & 805.11 & 45.00 & 46.73 & 45.87 \\
\hline 15 & Kolanka Goa & 34.75 & 39.64 & 37.19 & 1076.77 & 1011.74 & 1044.26 & 33.18 & 28.64 & 30.91 \\
\hline 16 & Kottapalli Kobbari & 21.75 & 27.94 & 24.84 & 1587.45 & 1536.43 & 1561.94 & 38.45 & 37.65 & 38.05 \\
\hline 17 & i Pasand & 20.90 & 24.84 & 22.87 & 1562.51 & 1502.32 & 1532.42 & 76.54 & 73.56 & 75.05 \\
\hline 18 & ndrews & 31.75 & 37.52 & 34.64 & 643.98 & 603.43 & 623.71 & 42.89 & 37.53 & 40.21 \\
\hline 19 & Nalla & 5.64 & 29.31 & & 1300.43 & 1267 & & 26.87 & & 25.40 \\
\hline 20 & Navaneetam & 21.25 & 27.42 & 24.33 & 898.66 & 842.32 & 870.49 & 34.65 & 31.92 & 33.28 \\
\hline 21 & Nuzividu Tiyy & & 32.74 & & 906.54 & & & 25.76 & & 23.93 \\
\hline 22 & du Rasalu & 22.25 & 28.94 & 25.59 & 1166.78 & 1019.73 & 1093.26 & 60.47 & 62.92 & 61.69 \\
\hline 23 & Panchadara Kalasa & 24.57 & 31.09 & & 976.98 & & & 21.23 & & 24.38 \\
\hline 24 & Pandurivari Mamidi & 25.62 & 34.87 & 30.24 & 1278.86 & 1174.35 & 1226.60 & 33.19 & 36.77 & 34.98 \\
\hline 25 & Paparao Goa & 43.67 & 41.65 & 42.66 & 925.76 & 910.22 & 917.99 & 98.30 & 97.77 & 98.04 \\
\hline 26 & Pedda & 25.21 & 36.84 & 31.02 & 978.76 & 932.54 & 955.65 & 25.77 & 22.87 & 24.32 \\
\hline 27 & Panukula Mamidi & 39.19 & 41.75 & 40.47 & 1256.66 & 1203.43 & 1230.05 & 23.98 & 20.87 & 22.42 \\
\hline 28 & Royal Special & 69.23 & 67.93 & 68.58 & 997.45 & 834.12 & 915.79 & 31.92 & 28.86 & 30.39 \\
\hline 29 & Rajamanu & 43.25 & 42.12 & 42.69 & 1324.64 & 1302.11 & 1313.38 & 28.55 & 25.55 & 27.05 \\
\hline 30 & Sora Mamidi & 25.25 & 26.74 & & 1253.63 & 1211.83 & 1232.73 & 19.93 & 19.87 & 19.90 \\
\hline 31 & Suvarnarekha & 26.75 & 31.31 & 29.03 & 1398.54 & 1324.73 & 1361.64 & 64.02 & 62.23 & 63.13 \\
\hline 32 & Tella Gulabi & 25.88 & 29.06 & 27.47 & 1464.87 & 1427.54 & 1446.21 & 23.90 & 25.85 & 24.88 \\
\hline 33 & Tella Rasalu & 22.25 & 28.53 & 25.39 & 996.56 & 983.28 & 989.92 & 75.56 & 71.12 & 73.34 \\
\hline 34 & Rajamamidi & 25.25 & 30.18 & 27.72 & 1043.21 & 1019.92 & 1031.56 & 30.34 & 26.63 & 28.49 \\
\hline & Mean & 30.67 & 34.48 & 32.57 & 1135.47 & 1087.51 & 1111.49 & 47.54 & 46.01 & 46.78 \\
\hline & SEm \pm & & & 0.81 & & & 37.64 & & & 0.94 \\
\hline & C.D 5\% & & & 2.26 & & & 105.09 & & & 2.62 \\
\hline & C.D $1 \%$ & & & 2.98 & & & 138.71 & & & 3.46 \\
\hline
\end{tabular}




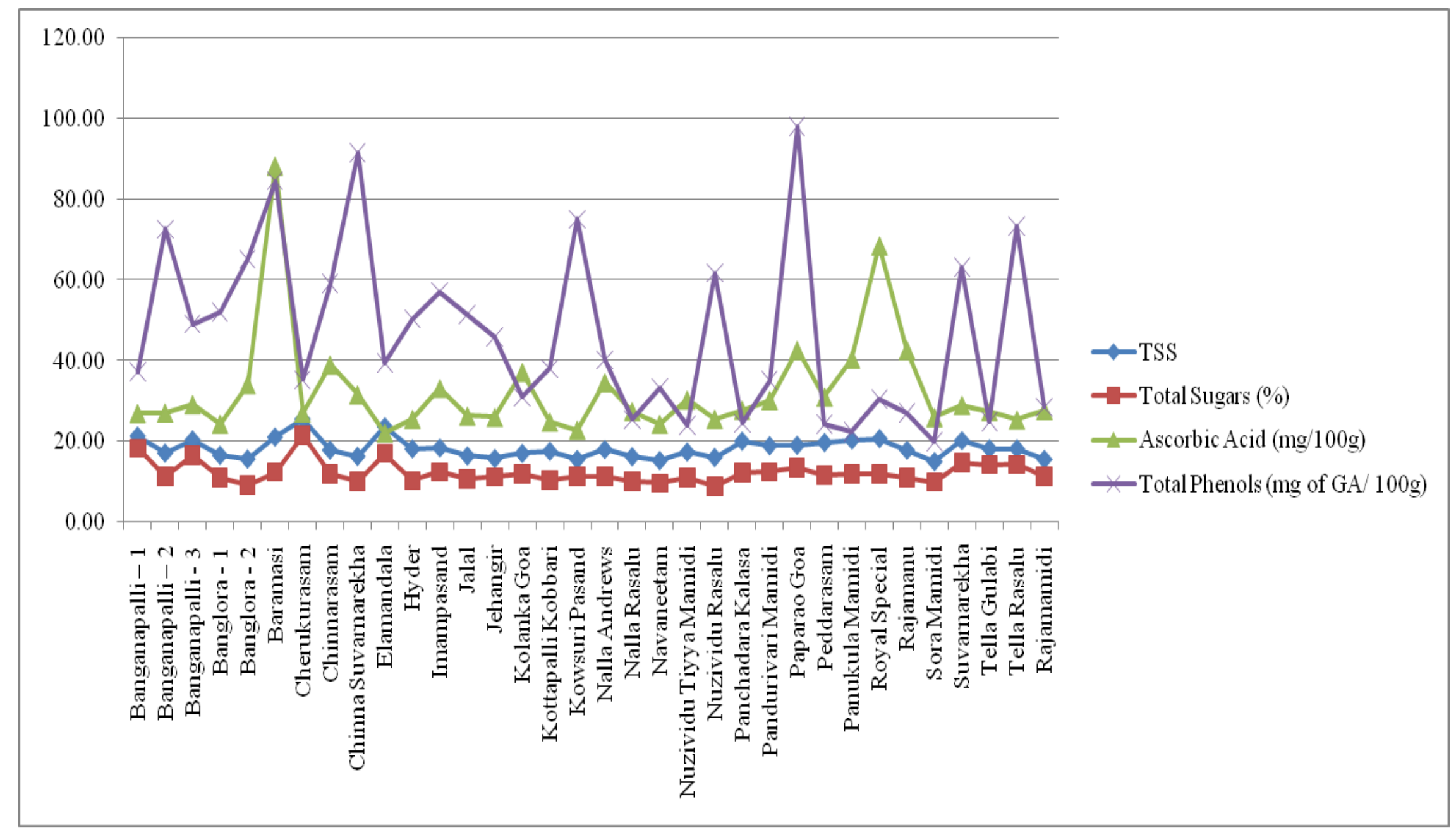

Fig. 1: Mean performance of mango cultivars for fruit bio-chemical characters 
Maximum non-reducing sugar content was found in Cherukurasam (15.39\%) followed by Banganapalli-1 (11.84\%) which was on par with Elamandala (11.26\%). Minimum nonreducing sugar content was found in Nuzividu Rasalu (5.80\%), which was on par with Hyder (6.22\%), Rajamanu (6.24\%), Chinna Suvarnarekha $(6.40 \%)$ and Navaneetham $(6.41 \%)$. The cultivars, Banganapalli-1, Banganapalli-3, Cherukursam, Elamandala, Suvarnarekha, Tella gulabi and Tella Rasalu having maximum total sugar and reducing sugar content can be used as potential parents for hybridization programme.

The predominant antioxidants in mango pulp are carotenoids (mainly $\beta$-carotene), ascorbic acid and total phenols. From the table 3 and figure 1, the highest ascorbic acid content was found in Baramasi (88.40\%) followed by Royal Special (68.58\%), Paparao Goa (42.66\%). Further, Panukula Mamidi $(40.47 \%)$ was on par with Paparao Goa. While, the lowest ascorbic acid content was found in Elamandala (22.14\%) followed by Kottapalli Kobbari (24.84\%). Further, the cultivars, Kowsuri Pasand (22.87\%), Nuzividu Tiyya Mamidi (24.33\%) and Banglora-1 (24.34\%) were on par with Elamandala. The results were in agreement with Rathor (2005) who stated that smaller sized mango fruits recorded higher ascorbic acid content than larger sized fruits. Fruit carotenoid is one of the most important parameters attributing yellow to orange color in pulp, the greater the carotenoids, the better the quality of the fruit. The $\beta$-carotene content ranged from 623.71 to $1679.89 \mu \mathrm{g} / 100 \mathrm{~g}$ with a mean value of $1111.49 \mu \mathrm{g} / 100 \mathrm{~g}$ (Table 3). Maximum $\beta$-carotene content was found in Chinnarasam $(1679.89 \mu \mathrm{g} / 100 \mathrm{~g})$ which was on par with Banganapalli-1 (1670.15 $\mu \mathrm{g} / 100 \mathrm{~g})$ and Hyder $(1668.49 \mu \mathrm{g} / 100 \mathrm{~g})$ while, minimum $\beta$-carotene content was found in Nalla Andrews $(623.71 \mu \mathrm{g} / 100 \mathrm{~g}$ ) which was on par with Baramasi (703.24 $\mu \mathrm{g} / 100 \mathrm{~g})$. From the results presented above, it was found that mango cultivars with yellow colored pulp were having maximum $\beta$ carotene content than mango cultivars with white colored pulp. More carotenoid content is known to result in dark orange coloured flesh which is a feature of consumer's preference. These genotypes may be considered promising as genitors for breeding programmes. The mean value of total phenol content was $46.78 \mathrm{mg} / 100 \mathrm{~g}$ while, it ranged from 19.90 to $98.04 \mathrm{mg} / 100 \mathrm{~g}$ (Table 3 and Fig.1). Approximately, the same trends observed for the ascorbic acid, relative to cultivar type, also occurred in the values for total phenol content measurements (Samal et al., 2012). The highest total phenol content was found in Paparao Goa (98.04 mg/100g) followed by Chinna Suvarnarekha (91.43 $\mathrm{mg} / 100 \mathrm{~g}$ ) and Baramasi (84.60 mg/100g) which recorded higher ascorbic acid content. The lowest total phenol content was found in Sora Mamidi (19.90 mg/100g) which was on par with Panukula Mamidi (22.42 mg/100g) which showed lower ascorbic acid content. An identical result with respect to total phenol content is reported by John et al., (2009) in mango. The cultivars Baramasi and Paparao Goa were extraordinarily high in ascorbic acid and total phenols compared to the other cultivars.

Similarly, the present study revealed some indigenous cultivars with superior fruit quality characters. In the present study, the selected cultivars expressed variable biochemical properties. Of the 34 mango cultivars subjected to bio-chemical characterization and evaluation, few were found to be horticulturally superior with respect to fruit bio-chemical characteristics. Fruit quality characterization and their evaluation are the prerequisites for proper conservation and efficient utilization in improving the better cultivars. These local promising germplasm can be used as potential 
parents for hybridization program for development of new hybrids.

\section{References}

AOAC, 1965. Association of Official Analytical Chemists. Official methods of analysis, AOAC, Washington D.C.

De-Candolle, A.D.E. 1904. Origin of Cultivated Plants. Kegan Paul, London.

Hammedunnisa Begum, Medagam Thirupathi Reddy, Surapaneni Malathi, Boreddy Purushotham Reddy, Sunil Arcahk, Javargowda Nagaraju and Ebrahimali Abubaker Siddiq. 2013. Morphological and Microsatellite Analysis of Intravarietal Heterogeneity in 'Beneshan' Mango (Mangifera indica L.). International Journal of Biotechnology Research and Practice. 1(1): 1-18.

John, A., Manthey, I. and Penelope, P. 2009. Levels of B-Carotene, Ascorbic Acid, and Total Phenols in the Pulp of Five Commercial Varieties of Mango (Mangifera indica L.). Proceedings of Florida State Horticultural Society 122:303-307.

Kulkarni, V. and Rameshwar, A. 1981. Biochemical and physical composition of fruits of some important Indian mango cultivars. Progressive Horticulture. 13(3-4): 5-8.

Malik, C.P., and Singh, M.B. 1980. In: plant enzymology and histo enymology. Kalyani Publishers, New Delhi. P. 286.
Mukherjee, S.K. 1950. Mango: Its allopolyploid nature. Nature. 166: 196197.

NHB, 2013. Indian Horticulture Database, National Horticulture Board, Guargon, India. $\quad h t t p: / / w w w . n h b . g o v . i n / a r e a$ production.html

Ranganna, S. 1986. Handbook of analysis and quality control for fruits and vegetable products. Tata Mc Graw Hill Publishing Company Limited, New Delhi.

Rathor, C.S. 2005. Genetic characterization of mango (Mangifera indica L.) germplasm under North Indian conditions. Part of M.Sc thesis submitted to the Indian Agricultural Research Institute.

Samal, K.C., Jena, R.C., Swain, S.S., Das, B.K. and Chand, P.K. 2012. Evaluation of genetic diversity among commercial cultivars, hybrids and local mango (Mangifera indica L.) genotypes of India using cumulative RAPD and ISSR markers. Euphytica. 185 (2): 195-213.

Simi, S. 2006. Characterization of traditional mango (Mangifera indica L.) varieties of southern Kerala Part of Ph. D Thesis submitted to the Kerala Agricultural University.

Srivastava, R.P and Sanjeev, K. 2014. Fruit and vegetable preservation principles and practices, third edition, Kalyani publishers, New Delhi.

\section{How to cite this article:}

Himabindu, A., D. Srihari, M. Rajasekhar, V. Sudhavani, P. Subbarammamma and Uma Krishna, K. 2018. Characterization and Quality Assessment of Potential Indigenous Mango (Mangifera indica L.) Cultivars of Coastal Districts in Andhra Pradesh, India by Bio-Chemical Markers. Int.J.Curr.Microbiol.App.Sci. 7(05): 2141-2151. doi: https://doi.org/10.20546/ijcmas.2018.705.249 